\title{
Oblique-incidence spatially resolved diffuse reflectance spectroscopic diagnosis of skin cancer
}

Alejandro Garcia-Uribe, Jun Zou, Tzu-Hsuan Chang, Madeleine Duvic, Victor Prieto, et al.

Alejandro Garcia-Uribe, Jun Zou, Tzu-Hsuan Chang, Madeleine Duvic, Victor Prieto, Lihong V. Wang, "Oblique-incidence spatially resolved diffuse reflectance spectroscopic diagnosis of skin cancer," Proc. SPIE 7572, Optical Diagnostics and Sensing X: Toward Point-of-Care Diagnostics, 75720L (25 February 2010); doi: 10.1117/12.842781

SPIE. Event: SPIE BiOS, 2010, San Francisco, California, United States 


\title{
Oblique-Incidence Spatially Resolved Diffuse Reflectance Spectroscopic Diagnosis of Skin Cancer
}

\author{
Alejandro Garcia-Uribe ${ }^{1,2}$, Jun Zou ${ }^{2}$, Tzu-Hsuan Chang ${ }^{2}$, Madeleine Duvic ${ }^{3}$, Victor Prieto ${ }^{3}$ and \\ Lihong V. Wang ${ }^{1}$ \\ ${ }^{1}$ Department of Biomedical Engineering, Washington University in St. Louis, \\ One Brookings Drive, St. Louis, Missouri, USA 63030 \\ ${ }^{2}$ Department of Electrical and Computer Engineering, Texas A\&M University, \\ 3128 TAMU, College Station, Texas, USA 77843 \\ ${ }^{3}$ Department of Dermatology, the University of Texas MD Anderson Cancer Center, \\ 1515 Holcombe Blvd., Houston, Texas, USA 77030
}

\begin{abstract}
This paper presents the use of spatially resolved oblique-incidence diffuse reflectance spectroscopy for skin cancer diagnosis. Spatio-spectral data from 166 pigmented skin lesions were collected for the wavelength range from 455 to $765 \mathrm{~nm}$. A set of neural network based classifiers separates the pigmented malignant melanomas from the benign and dysplastic subgroups. A total of 110 lesions were used as the training set and 56 lesions were used as the testing set. This classifier performs with an overall $100 \%$ sensitivity and $92 \%$ specificity for the training set and $100 \%$ sensitivity and $88 \%$ specificity for the testing set. The second classifier was designed to separate the benign from the dysplastic subgroups. For the second classifier a total of 100 lesions were used as the training set and 51 lesions were used as the testing set. The overall classification rates were $94 \%$ and $88 \%$ for the training and testing sets respectively.
\end{abstract}

Keywords: diffuse reflectance, spectroscopy, skin cancer, melanoma.

\section{INTRODUCTION}

Recent advancement in biomedical optics has revealed that the optical properties of human epithelial tissues are linked to certain critical physiological parameters of their cancer states ${ }^{1-4}$. Therefore, developing and applying novel optical sensing or imaging techniques to capture and process these optical parameters is expected to have profound medical significance in the diagnosis of various epithelial cancers. Skin cancer is the most common form of cancer, with about a million new cases estimated in the U.S. each year. Melanoma is the most serious type of skin cancer and is the leading cause of death among the skin diseases. Melanoma develops in the cells that produce melanin (melanocytes). The American Cancer Society estimates that every year there are more than 62,000 new cases of melanoma and about 8,400 people are expected to die of this disease in the United States. ${ }^{5}$ Early detection is a key factor for successful treatment of skin cancer. Our previous studies have shown that Oblique Incidence Diffuse Reflectance Spectrometry (OIDRS) as an effective tool to separate carcinoma skin cancer lesions from benign keratoses. ${ }^{6}$ In clinical practice, dermatologists use the $\mathrm{ABCD}$ rule (asymmetry, border, color and diameter) and change in the appearance of a mole or pigmented area, to spot suspicious skin lesions. After the skin check a skin biopsy is preformed for assessing whether the pigmented lesion is benign or malignant. Dysplastic nevi are atypical moles with architectural and/or cytological disorder. Depending of the degree of atypia, dysplastic nevi are mild moderate or severe. Researchers believe that dysplastic nevi are more likely than ordinary moles to develop into a melanoma. ${ }^{7}$ Actinic keratosis, seborrheic keratosis, compound and junctional nevi are common types of benign skin lesions.

\section{EXPERIMENTAL SETUP}

A halogen lamp is used as the light source. The optical multiplexer allows the light delivery through only one source fiber at a time to the area of interest. Once delivered to the skin, the incident light interacts with the medium and the

Optical Diagnostics and Sensing X: Toward Point-of-Care Diagnostics, edited by Gerard L. Coté,

Proc. of SPIE Vol. 7572, 75720L · C 2010 SPIE · CCC code: 1605-7422/10/\$18 · doi: 10.1117/12.842781 
diffuse reflectance is collected by the collection optical fibers. The collection fibers are coupled with the imaging spectrograph that generates an optical spectrum for each fiber. The CCD camera collects the spatially resolved diffused reflectance for the wavelength range from 455 to $765 \mathrm{~nm}$. Finally the information is storage in a personal computer for further analysis (Fig. 1). The probe consists of 5 source fibers and two linear arrays of twelve collection fibers within an area $2 \times 2 \mathrm{~mm}^{2}$ (Fig. 2 )

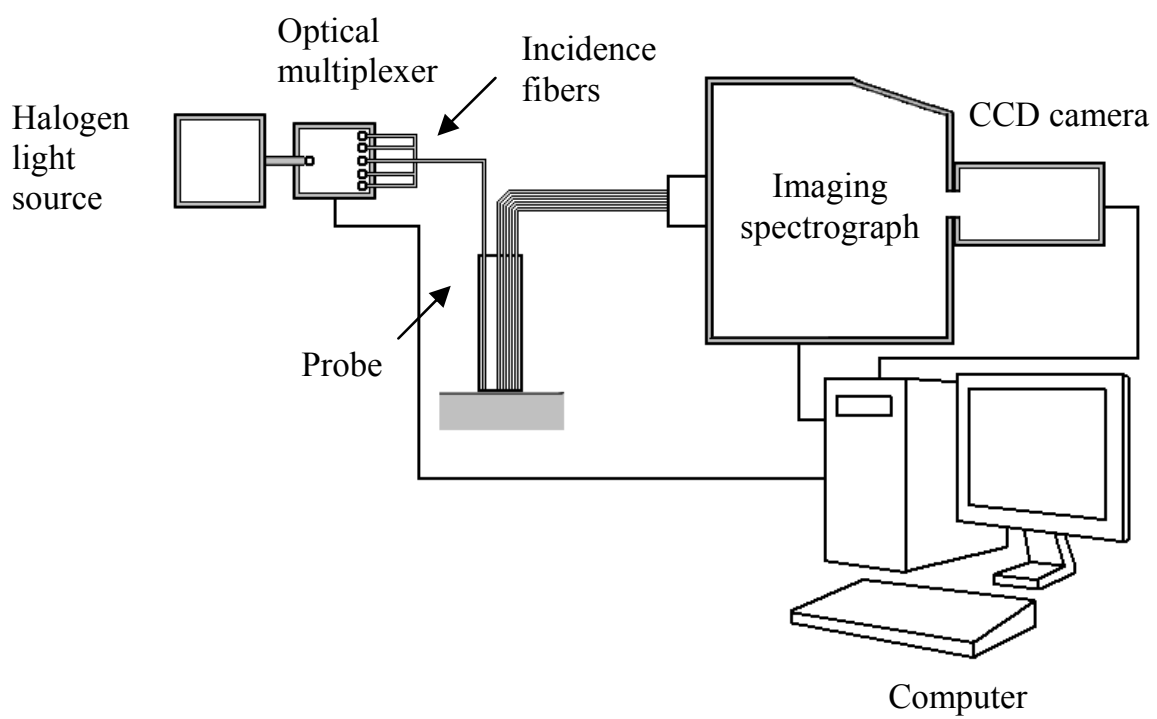

Fig. 1. System setup.

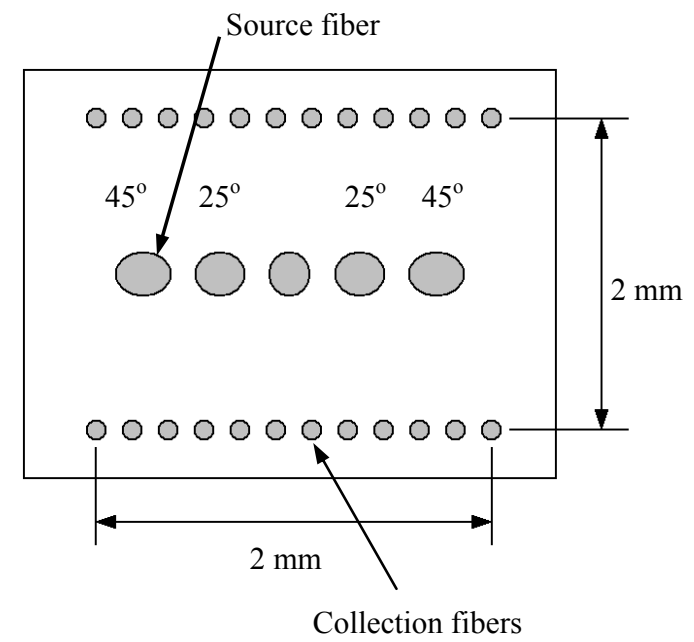

Fig. 2. Probe description.

\section{EXPERIMENTAL RESULTS}

The data collection was performed at the University of Texas M.D. Anderson Cancer Center in Houston. First, the physician would identify the lesion(s) of interest scheduled for biopsy. The measurement from each lesion was repeated four times, the probe was repositioned to obtain images from different locations and orientations to average out the effect of structural anisotropy. This process was repeated for the neighboring healthy skin. The spatially resolved 
diffuse reflectance spectra from 166 pigmented. Among the 166 skin lesion samples, there were 15 melanomas (M), 8 severe dysplastic nevi (DN3), 61 moderate dysplastic nevi (DN2), 30 mild dysplastic nevi (DN1), 31 common nevi $(\mathrm{CN}), 5$ actinic keratosis (AK) and 16 seborrheic keratosis (SK). The initial set of features is based on the wavelet transform and selected using the fisher distance between the two classes as a separability messurement. ${ }^{6}$ An artificial neural network based classifier is combined with a genetic algorithm to maximize the classification rate. Two thirds of the data set is used for training and one third is use for testing. A total of 110 lesions were used for training and 56 lesions were used for testing. As shown in Fig. 3. This classifier for the training data set performs with $100 \%$ sensitivity and $92 \%$ overall classification rate specificity. The Fig. 4 shows the scatter plot for the testing set with sensitivity of $100 \%$ and a specificity of $88 \%$. A second classifier is designed to distinguish the benign common nevi actinic keratosis and seborrheic keratosis from mild, moderate and severe dysplastic nevi. A total of 100 lesions were used for training and 51 lesions were for testing. The overall classification rates are $94 \%$ for the training set and $88 \%$ for the testing set.

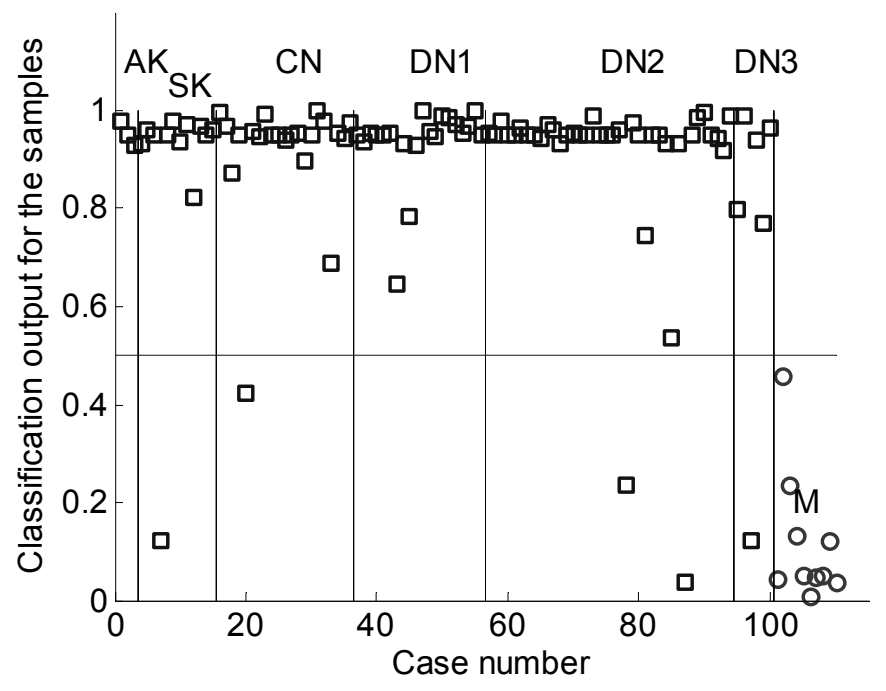

Fig 3. Scatter plot for the training data set.

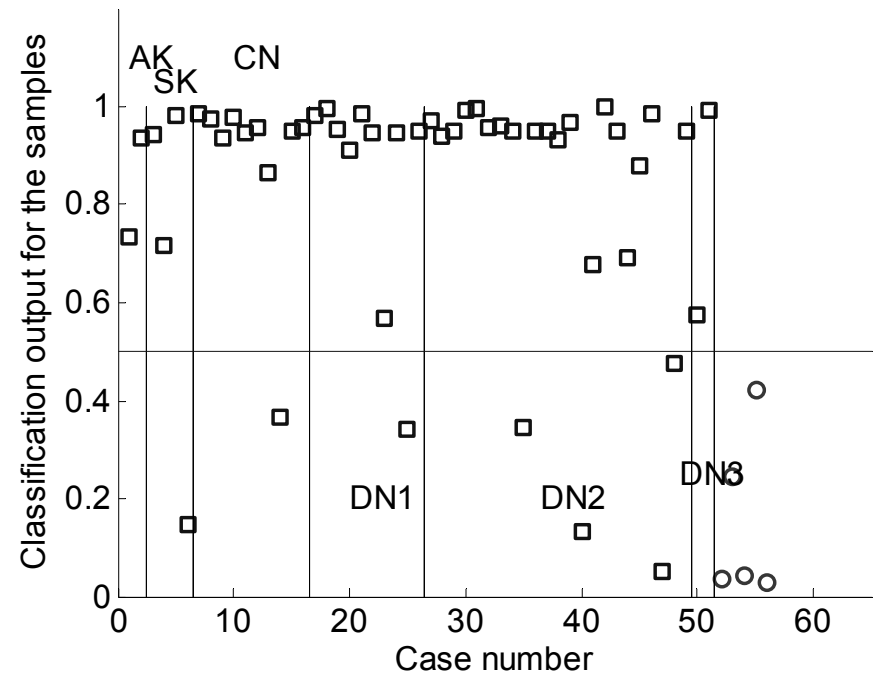

Fig 4. Scatter plot for the testing data set. 


\section{CONCLUSION}

An oblique incidence diffuse reflectance spectroscopy system has been proved to be an effective tool to differentiate pigmented benign, dysplastic, and malignant skin lesions. These results are highly promising and indicate that this approach, successfully used on skin, can potentially be used for other epithelial types of cancers.

\section{ACKNOWLEDGMENTS}

The authors thank Dr. Mays, Dr. Hymens and Dr. Mansfield at the University of Texas M. D. Anderson Cancer Center for their help during the data collection. This project is sponsored by National Institute of Health grant R01 CA106728.

\section{REFERENCES}

1. P. R. Bargo, S. A. Prahl., T. T. Goodell, R. A. Sleven, G. Koval, G. Blair, S. L. Jacques, "In vivo determination of optical properties of normal and tumor tissue with white light reflectance and an empirical light transport model during endoscopy", J. Biomedical Optics 10, 034018, 2005.

2. V. P. Wallace, J. C. Bamber, D. C. Crawford, R. J. Ott, and P. S. Mortimer, "Classification of reflectance spectra from pigmented skin lesions, a comparison of multivariate discriminant analysis and artificial neural networks," Phys. Med. Biol. 45, 2859-2871, 2000.

3. I. Georgakoudi, B. C. Jacobson, J. Van Dam, V. Backman, M. B. Wallace, M. G. Muller, Q. Zhang, K. Badizadegan, D. Sun, G. A. Thomas, L. T. Perelman, M. S. Feld, "Fluorescence, reflectance, and light-scattering spectroscopy for evaluating dysplasia in patients with Barrett's esophagus", Gastroenterology 120, 1620-1629, 2001.

4. Y. N. Mirabal, S.K Chang, E. N. Atkinson, A. Malpica, M. Follen, R. Richards-Kortum, "Reflectance spectroscopy for in vivo detection of cervical precancer," J. Biomedical Optics 7, 587-594, 2002.

5. American Cancer Society website, "Overview: skin cancer - Melanoma” Available: http://www.cancer.org.

6. A. Garcia-Uribe, N. Kehtarnavaz, G. Marquez, V. Prieto, M. Duvic, and L. V. Wang, "Skin cancer detection by spectroscopic oblique-incidence reflectometry: Classification and Physiological Origins," Appl. Opt. 43, 2643-2650, 2004.

7. M. L. Williams and R. W. Sagebiel, "Melanoma risk factors and atypical moles," West J Med. 160, 343-350, 1994. 